DOI: $10.1051 /$ epjconf / 20146702047

(c) Owned by the authors, published by EDP Sciences, 2014

\title{
The pressure gradient in the human respiratory tract
}

\author{
Michaela Chovancová ${ }^{1}$ and Jakub Elcner ${ }^{1}$ \\ ${ }^{1}$ Faculty of Mechanical Engineering, Brno University of Technology, Dept. of Themodynamics and Enviromental \\ Engineering, Technická 2896/2, 61669 Brno, Czech Republic
}

\begin{abstract}
Respiratory airways cause resistance to air flow during inhalation and exhalation. The pressure gradient is necessary to transport the air from the mount (or nose) to pulmonary alveoli. The knowledge of pressure gradient (i.e. respiratory airways resistance) is also needed to solve the question of aerosol deposition in the human respiratory tract. The obtained data will be used as boundary conditions for CFD simulations of aerosol transport. Understanding of aerosol transport in the human lungs can help us to determine the health hazard of harmful particles. On the other hand it can be used to set the conditions for transport of medication to the desirable place. This article deals with the description of the mathematical equations defining the pressure gradient and resistance in the bronchial three and describes the geometry used in the calculation.
\end{abstract}

\section{Introduction}

Understanding of the mechanism of the breathing is important for the prevention of the diseases and also for determine the method of the treatment that can cure these diseases. For this purpose it is necessary to have information on the transport and deposition of aerosols particles in the respiratory tract of humans. The obtained data will be used for computer model of airflow in the lungs during inspiration in CFD software (it can help us with identify the conditions which the medication should be transported to the desired location). One of the boundary conditions which is entered for the most realistic running of the simulation is the pressure gradient in the airways. It is required to ensure that the air can circulate between the mouth (or nose) and the pulmonary alveoli. The pressure gradient is formulated as the difference between alveolar and atmospheric pressure (in oral or nasal cavity is assumed atmospheric pressure). During inspiration is the alveolar pressure less than atmospheric (negative intrapleural pressure produces expansion the volume of the lungs and the result of that is pressure drop in the lungs and develop negative alveolar pressure). During exhalation is alveolar pressure upper than atmospheric (when inspiration muscles relax than elasticity of lungs dominates and the thoracic volume and lungs volume decreases and alveolar pressure becomes positive).

Pressure drop is directly relates with the resistance of the airways. Airways resistance is the opposition to flow caused by the forces of friction. It is defined as the ratio of driving pressure to the rate of air flow. Resistance to flow in the airways depends on whether the flow is

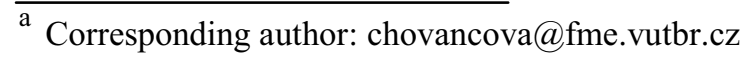

laminar or turbulent, on the dimensions of the airway and on the viscosity of the gas [10].

\section{Morphology of the lungs used in the calculation}

To determine the pressure drop of the airways (and with it associated resistance) is primary define their geometry.

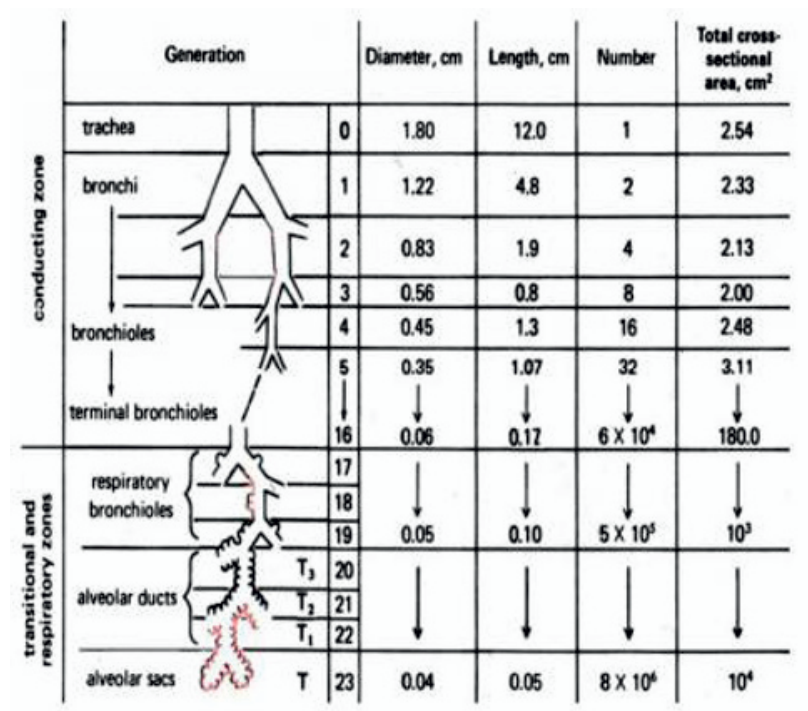

Figure 1. Diving of the bronchial tree.

The airways begin in nasal or oral cavity where air flows in to the pharynx. The pharynx is divides into three parts, namely the nasopharynx, oropharynx and 
laryngopharynx. The laryngeal part passes into the larynx. It is the boundary between the upper and lower airways. Trachea connects to the larynx. The air passes through the trachea and purified there by cilia that trap it dirt. The trachea enters to the chest where it branches into right and left bronchi. The bronchi changes after third generation of the branching to the bronchioles. Bronchial tree totally consists of 23 generations. From the trachea to $16^{\text {th }}$ generation is conductive part, from $17^{\text {th }}$ to $19^{\text {th }}$ generation is respiration part where is partially exchanged air. $20^{\text {th }}$ generation of the branching includes of the alveolar ducts and they are terminated with alveolar sacs ( $23^{\text {th }}$ generation). The alveolar sacs contain the alveoli. There are passed diffusion of the gas between blood and air $[3,11,13]$.

On figure 2 we can see the real geometry of the bronchial tree. It is complicated and disparate. Any way from mouth to alveoli is the same. As the number of the alveoli of the adult males ( 30 years old) is around 310 million, that would means to calculate pressure drop for the 310 million ways.

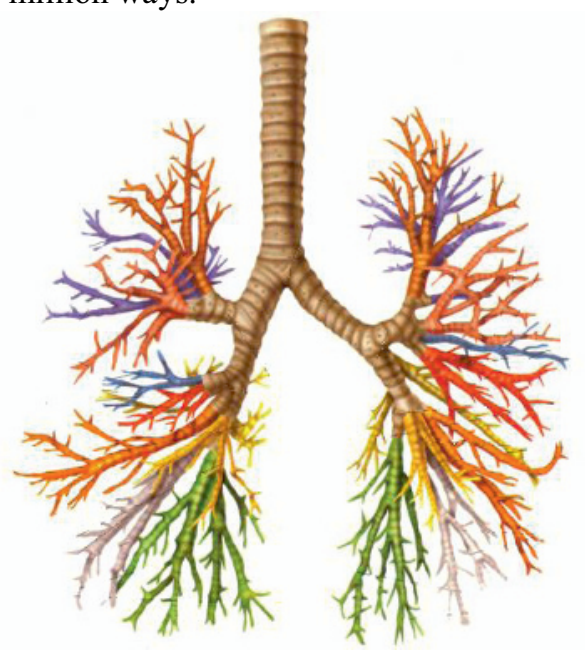

Figure 2. Bronchial tree.

Therefore is chosen for the calculation the Weibel's idealized model of the bronchial tree. It is symmetric model (all ways to alveoli is the same) and is based on the measured values, which were evaluated by statistical methods.

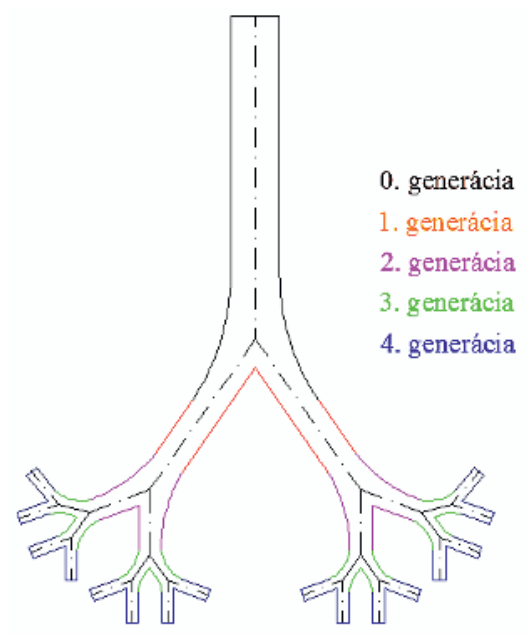

Figure 3. Weibel's idealized model of the bronchial tree.
The proportions of Weibel's lungs (WL) are dimensions of prepared lungs of the adult male. The lungs are inflated to the volume of $4800 \mathrm{ml} \mathrm{(3/4} \mathrm{to} \mathrm{its}$ total volume). It are the static dimensions of the lungs with total lung capacity (TLC) $6400 \mathrm{ml}$. For the total lung capacity is residual volume around $2600 \mathrm{ml}$. The pressure drop are solved for quit inspiration with $500 \mathrm{ml}$ tidal volume (VT) and for forced inspiration (FI) with $2200 \mathrm{ml}$ tidal volume. After forced inhalation is lung volume and the diameters of the airways the same as Weibel's volume.

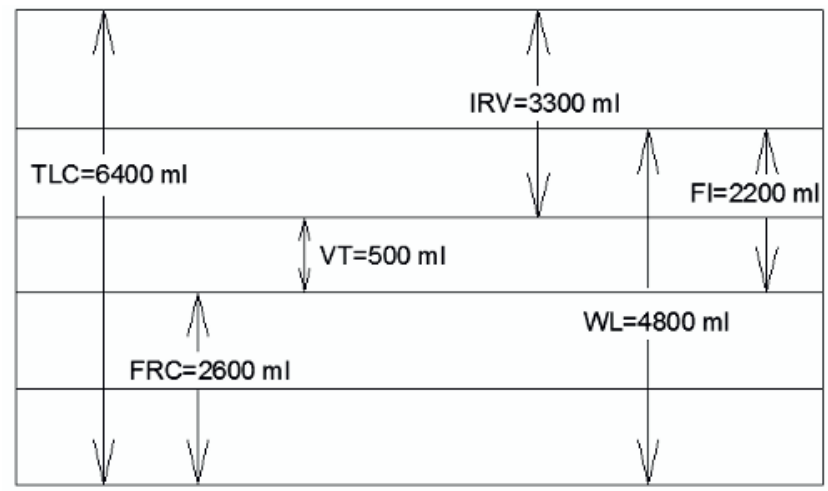

Figure 4. Capacity of Weibel's lungs.

The dimensions of the airways when the lung has FRD volume (sum of the air volume which can be still exhaled after quit expiratory and air volume residual in the lungs after maximum exhalation), ergo at the beginning resting of the respiratory cycle, was determined by taking away the volume $2200 \mathrm{ml}$ from Weibel's lungs. It is assumed that the each generation in the bronchial tree have the same elasticity besides trachea and primary bronchi. There is assumed zero elasticity given to the presence of the cartilages. Then the volume of the each generation is determined like percentage of Weibel's lungs. So the final volume of the each generation at the beginning of the inspiration is $46 \%$ of the volume the each generation of the Weibel's model.

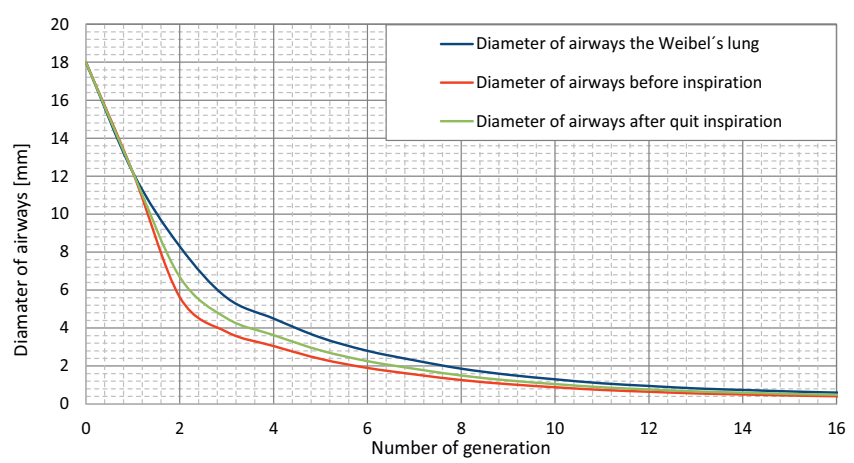

Figure 5. Dimensions of lung at the beginning and at the end of the inspiration.

During the inspiratory and the expiratory is changed mainly the cross-section. Their length change just very little and has not effect on the airways resistance, therefore this change is not considered in the calculation. 
Than the cross-sectional of the airways before inspiration is simple determined from the volume of the airways at the beginning of the respiratory cycle. Similarly are determined the dimensions of the airways with lung volume $3100 \mathrm{ml}(\mathrm{FRC}+\mathrm{VT}=$ volume of the lungs after resting inspiration).

\section{Method of the calculation}

As in the classic pipe systems is the pressure loss in the bronchial tree caused by friction forces and the local resistance in the bifurcation. The solving of the pressure drop the respiratory tract is more complicated because the airways are flexible.

Mathematical formula for the calculation the pressure gradient of the air flow through tube of the length $\mathrm{L}$ and diameter $r$ is determined Poiseuille's law:

$$
\Delta \mathrm{P}_{\mathrm{d}}=\frac{8 \mathrm{~L}}{\pi r^{4}} \cdot \mu \cdot \dot{\mathrm{V}}
$$

Mathematical equation 1 is valid for laminar airflow in the bronchial tree. During resting breathing with airflow $15 \mathrm{l} / \mathrm{min}$ and tidal volume 0.51 is there laminar flow. The maximum value of the Reynolds number is 1130. During forced inspiration with the airflow $60 \mathrm{l} / \mathrm{min}$ and tidal volume 2.21 arises in the first three generation the turbulent flow. They are designed with the mathematical equation for the calculation pressure loss over for the turbulent flow in the pipe

$$
\Delta P_{d}=\lambda \cdot \frac{L}{d} \cdot \frac{v^{2}}{2} \cdot \rho
$$

where frictional factor are calculated by Blasius's empirical relationship.

The local pressure loss, thus the loss incurred by change of the cross-sectional $\left(\mathrm{A}_{1} \rightarrow \mathrm{A}_{2}\right)$ which overflow:

$$
\Delta \mathrm{P}_{\mathrm{m}}=\frac{\rho}{2 \cdot \mathrm{C}^{2}} \cdot\left(\frac{1}{\mathrm{~A}_{2}^{2}}-\frac{1}{\mathrm{~A}_{1}^{2}}\right) \cdot \dot{\mathrm{V}}^{2}
$$

The equation is derived from the Bernoulli equation (equation of the conservation of the mechanical energy). Conversion factor $\mathrm{C}$ taken into account the branching angle and Reylnold's number and its value is around $0.6-0.7$. $[1,6,7,8,9]$

Both turbulent and laminar flow cause resistance to air moving in the airways (exactly for its overcome is needed the pressure gradient). The airways resistance are measured in $\mathrm{cmH}_{2} \mathrm{O} / \mathrm{l} / \mathrm{s}$ and is defined as the ratio of the pressure difference to the airflow.

$$
R=\frac{\Delta P}{\dot{V}} \quad\left(\mathrm{~cm} \mathrm{H}_{2} \mathrm{O} / 1 / \mathrm{s}\right) .
$$

For total airways resistance is pressure drop the difference between mouth and the alveoli.
The value of the airways resistance during the resting breathing is around $1.5-2 \mathrm{~cm} \mathrm{H}_{2} \mathrm{O} / 1 / \mathrm{s}$. One would expect the major site of resistance, based on the Poiseuille law to be located in the narrow airways (the bronchioles), which have the smallest radius. However, measurements show that only $10 \%$ to $20 \%$ of total airway resistance can be attributed to the small airways (those $<$ $2 \mathrm{~mm}$ in diameter). This apparent paradox results because so many small airways are arranged in parallel and their resistances are added as reciprocals. Resistance of each individual bronchiole is relatively high, but the great number of them results in a large total cross sectional area, causing their total combined resistance to be low $[2$, $4,5,6]$.

In a healthy person has a major influence on the airways resistance the lung volume change (generally when lung volume increases resistance decreases) and breathing mode change thus its flow.

\section{Results}

Calculations are solved for two breathing mode:

$\begin{array}{rlrl}\text { Q) } & & =151 / \mathrm{min} \\ \text { b) } & \text { tidal volume } & =0.51 \\ & & \text { airflow } & =601 / \mathrm{min} \\ \text { tidal volume } & =2.21\end{array}$

During the quiet inspiration the airflow has the laminar character through whole airways. On the solution of the linear pressure losses in the laminar flow is used equation 1. Volumetric flow in the individual parts of the respiratory tract is determined by the continuity equation.

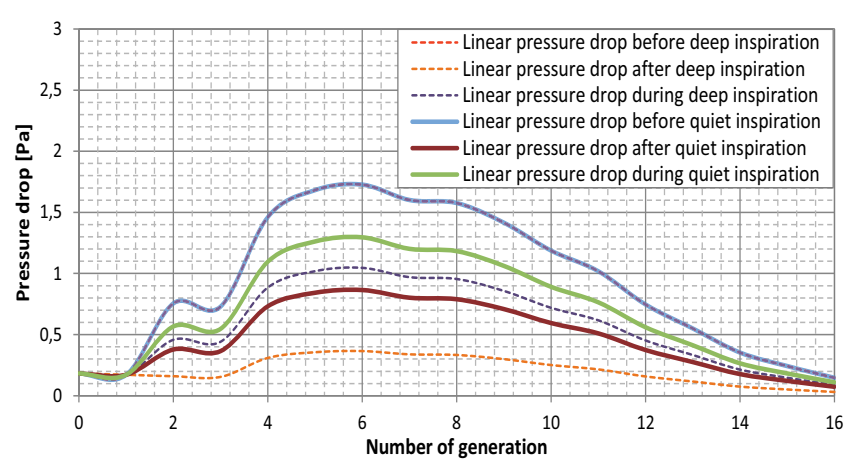

Figure 6. Linear pressure drop in the airways during quiet inspiration and deep inspiration $(\mathrm{VT}=2.21$ and flow $=$ $151 / \mathrm{min})$.

The linear pressure losses before inhalation are determined from lungs static dimensions before breathing cycle. Similarly are determined the losses after inhalation (lung static dimensions after inhalation). It can be assumed that the linear pressure drop during inspiration will be found between the curve of the pressure losses before inhalation (the smallest cross-sectional of the airways during respiration, it means the most pressure losses) and curve of the pressure losses after the inhalation (the largest cross-sectional, it means the smallest pressure losses). In our calculations are determined it as average value of the linear pressure losses before and after breathing (figure 6.). 
For the comparison of influence change the cross section of airways is found in the graph also the linear pressure losses for deep inspiration thus with the same airflow $(15 \mathrm{l} / \mathrm{min})$ but with the tidal volume 2.21 . The graph show that with increasing of the lung volume the linear pressure drop decreases. The changing the pressure losses affected only by the volume changing is not so pronounced. The greatest linear pressure drop is located in the $6^{\text {th }}$ generation and from that point it decreases.

In the evaluation the pressure drop of the airways can't forget to the local pressure drop, which also in classic pipe systems represent a major proportion of the total pressure losses. They are determined by the mathematical equation 3. For inhaled and exhaled static lungs are installed in the mathematical equation the crosssection consecutive generation and associated values of the airflow flowing into next generation. When calculating local pressure drop during inspiration it is considered that the increase of the airways is steady this means that the local pressure drop during the inspiration will be in the range between the locale pressure loss before inhalation and after inhalation. In our calculations are determined their average value.

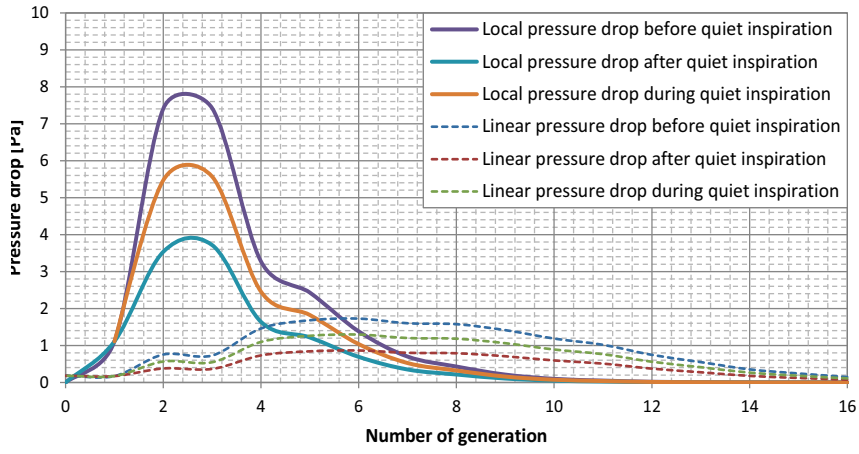

Figure 7. Local and linear pressure drop in the airways during quit inhalation

Figure 7. shows the performance of the local and linear pressure drop for quiet breathing. On the graph can be seen that also local pressure drop decrease with increasing of the volume. The value of the local pressure drop decreases significantly in the $9^{\text {th }}$ generation. There is less significant changes between cross-section of adjacent generation and flows through them less airflow. From $6^{\text {th }}$ generation it has smaller value like linear pressure drop.

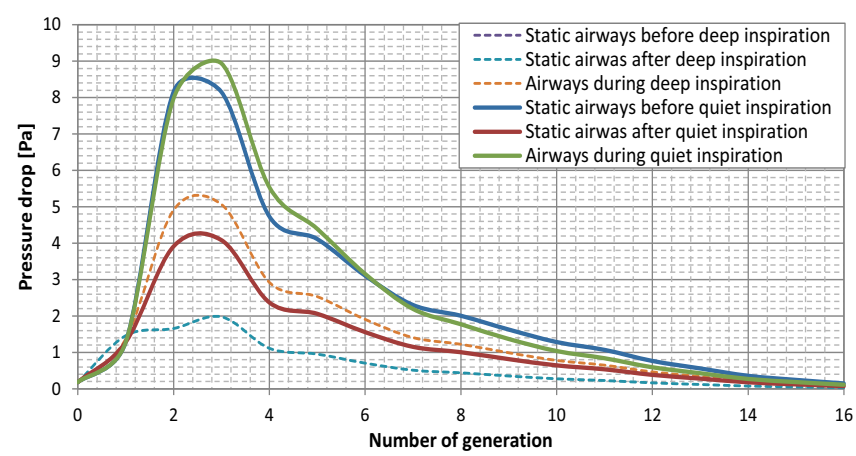

Figure 8. Total pressure drop in the airways during quiet inhalation and deep inhalation (VT $=2.21, \dot{V}=151 / \mathrm{min}$ ).
The total pressure drop of the airways (figure 8.) is the sum of the local and linear pressure losses. Figure 8 . shows influence of the volume change on the total pressure drop of the airways. It decreases with increasing volume. The changing the total pressure losses affected only by the volume changing is not so pronounced. Its value during quiet inhalation in the conductive part of the airways is quantified to $31 \mathrm{~Pa}$ and its corresponding value of the airways resistance is $1.2 \mathrm{~cm} \mathrm{H}_{2} \mathrm{O} / 1 / \mathrm{s}$.

The calculations of the forced breathing is the same like a calculation of the quiet breathing but the dimensions of the airways in the end of inhalation have dimensions Weibel's lungs and the airflow is $601 / \mathrm{min}$. The flow rate with this value causes in the first three generations the turbulent flow. There are calculated the linear pressure drop by equation 2 . In this area can be seen the highest value and the highest increase compared to the quiet breathing.

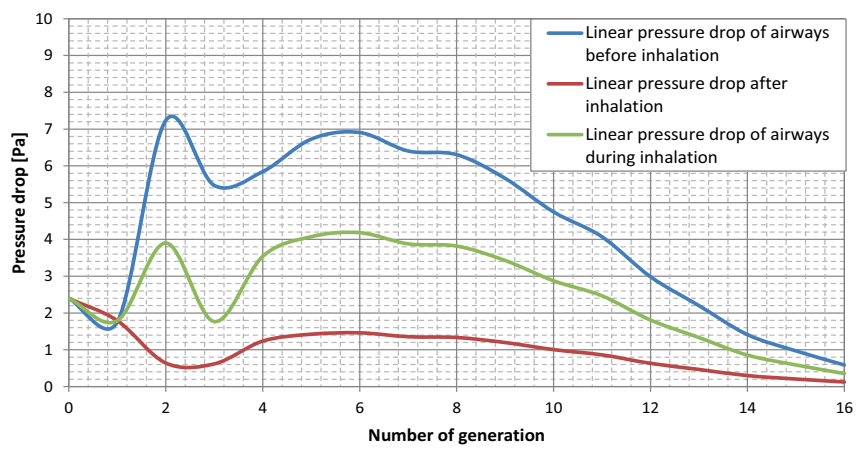

Figure 9. Linear pressure drop in the airways during forced inhalation

The linear pressure losses are more pronounced in the area with the turbulent flow. The highest value is in the $2^{\text {nd }}$ generation. In the laminar region has the top values in the $6^{\text {th }}$ generation and from that point it decreases.

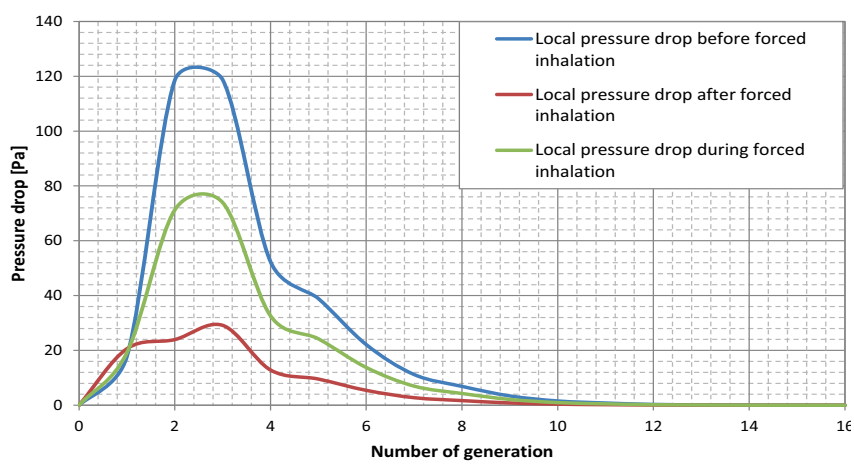

Figure 10. Local pressure drop in the airways during forced inhalation

Local pressure losses have significant growth in the first nine generations. From $10^{\text {th }}$ generation are gradually approached to the values during the quiet breathing. 


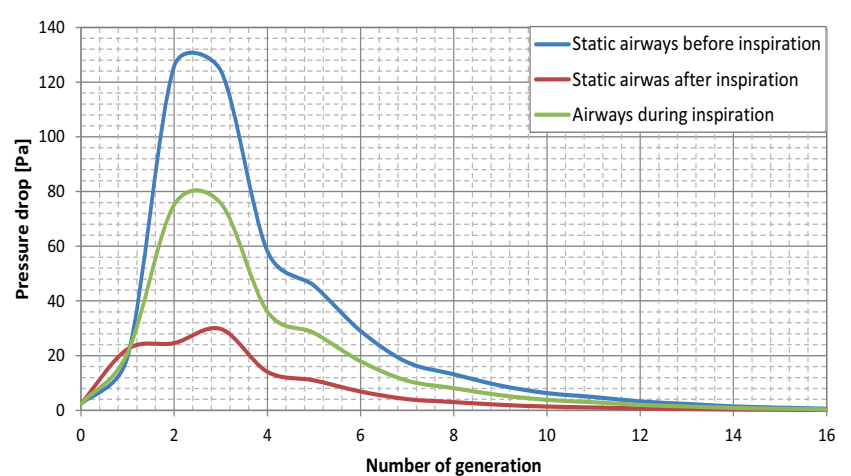

Figure 11. Total pressure drop in airways during forced inhalation

The highest loss is in the $3^{\text {th }}$ generation. The total pressure lost during forced inhalation increased to $293 \mathrm{~Pa}$ and its corresponding value of the airways resistance is $3 \mathrm{cmH}_{2} \mathrm{O} / \mathrm{l} / \mathrm{s}$.

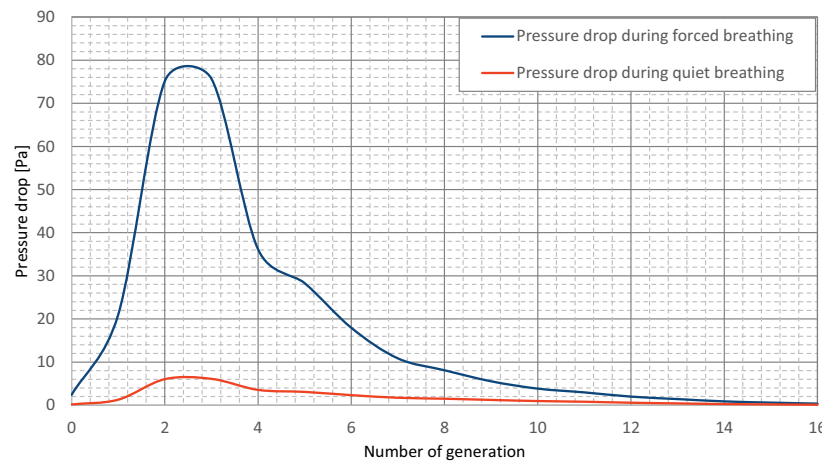

Figure 12. Pressure drop in airway

Airway pressure losses does not change much during normal quiet breathing. It is significantly increased, however, during forced expiration. The increase of the pressure losses during forced inhalation compared the quit breathing is significant, but the differences begin to lose around $14^{\text {th }}$ generation. The value of the pressure loss in the lower generations is relative small and don't grow significant neither volume change nor change of airflow.

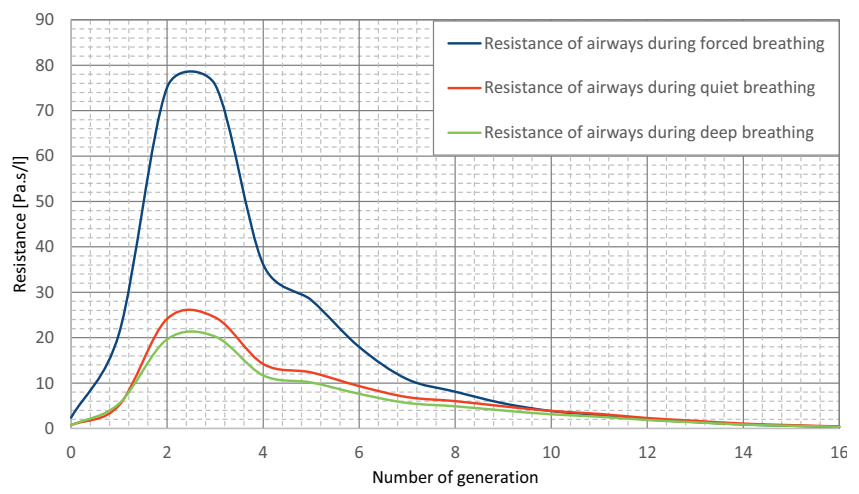

Figure 13. Resistance of airways

The resistance becomes highest in larger airways and as the total cross sectional area of the airways increase with branching of the airways, the resistance diminishes. The difference airways resistance during forced inhalation and quit inhalation and deep inhalation is significant mainly in the initial generations. On the $9^{\text {th }}$ generation differences begin to lose.

\section{Conclusion}

In such a complicated system of tubes as the bronchial tree with its many branches, changes in caliber and irregular wall surfaces, the application of the above principles is difficult. In practice entrance conditions of the tube are critical. Our simplified calculations approximate to real values of the total resistance in the lung during quit inspiration so we also considered the other results approximating to true value. These calculations of pressure losses and resistances will help us to a more accurate setting of boundary conditions for simulation the respiratory process in the CFD software and will help us solved aerosol deposition in the respiratory tract of humans.

\section{Acknowledgement}

This article was supported by the project Excellent young researcher at BUT (CZ.1.07/2.3.00/30.0039)

\section{References}

1. W. O. Feen, H. Rahn, Handbook of Physiology: Respiration (American Physiological Society, Washington, D.C., 1964)

2. R. A. Rhoades, D. R. Bell., Medical Physiology (Lippincott Williams and Wilkins, Philadalphia, 2008)

3. E. R. Weibel, Morphometry of the human lung (Springer - Verlag, Berlin, 1963)

4. R. M. Schwartzstein, M. J. Parker, Respiratory physiology: A clinical approach (Lippincott Williams and Wilkins, Philadalphia, 2006)

5. J. B. West, Respiratory physiology: The essentials (Lippincott Williams and Wilkins, Philadalphia, 2008)

6. A. Ben-Tal, Simplified models for gas exchnage in the human lungs, J. of Theor. Biology, 474 (2005)

7. S. Kobashi, K. Kuramoto, Y. Hata, Funct. assessment of individual lung lobes with MDCT images, Intech, 96 (2011)

8. V. K. Verma, V. K. Katiyar, M. P. Singh, Study of periodic breathing and human respiratory system, In. J. of Medicne and Medicla Sc, 330 (2009)

9. F. S. Grodins,J. Buell, A. J. Bart, Mathematical analysis and digital simulation of the respiratory control system. J. Appl. Physiol. 260 (1967)

10. E. P. Hill, G. G. Power, L. D. Longo, Mathematical simulation of pulmonary $\mathrm{O} 2$ and $\mathrm{CO} 2$ exchange. Am. J. Physiol. 904 (1973) 
11. F. G. Hoppin Jr., J. Hildebrandt, Mechanical properties of the Lung. In: (West, J.B. (Ed.), New York, 1977)

12. E. R. McFadden Jr., Respiratory heat and water exchange: physiological and clinical implications. J. Appl. Physiol., 331 (1983)

13. D. W. Carley, D. C. Shannon, A minimal mathematical model of human periodic breathing. J. Appl. Physiol. 1400 (1988) 